\title{
miRNA expression profiling regulates necroptotic cell death in hepatocellular carcinoma
}

\author{
MARIA VISALLI $^{1}$, MARCELLO BARTOLOTTA $^{2}$, FRANCESCA POLITO $^{1}$, ROSARIA OTERI $^{1}$, \\ ADALBERTO BARBERA ${ }^{2}$, ROBERTO ARRIGO ${ }^{1}$, ROSA MARIA DI GIORGIO ${ }^{1}$, \\ GIUSEPPE NAVARRA ${ }^{2}$ and M'HAMMED AGUENNOUZ ${ }^{1}$
}

\author{
${ }^{1}$ Functional Genomics Laboratory, UOC Neurologia MNM, Department of Clinical and Experimental Medicine, \\ University of Messina; ${ }^{2}$ Oncology Surgery Unit, Department of Human Pathology DETEV, \\ University of Messina, I-98125 Messina, Italy
}

Received January 12, 2018; Accepted April 10, 2018

DOI: $10.3892 /$ ijo.2018.4410

\begin{abstract}
Hepatocellular carcinoma (HCC) is one of the most aggressive types of cancer and is among the leading causes of cancer-related mortality worldwide. Although the dysregulation of microRNAs (miRNAs or miRs) has often been reported in HCC, the precise molecular mechanisms by which miRNAs modulate the process of tumorigenesis and the behavior of cancer cells are not yet clearly understood. In this study, we identified a novel three-miRNA signature, including miR-371-5p, miR-373 and miR-543, that appears to orchestrate programmed cell necrosis in HCC by directly targeting the caspase- 8 gene (Casp-8). Our results demonstrated that miR-371-5p, miR-373 and miR-543 were overexpressed in HCC tissues compared with paired adjacent normal tissues. The upregulation of these miRNAs specifically and markedly downregulated the expression of Casp-8, as well as significantly enhanced the Z-VAD/TNF- $\alpha$-induced necroptosis of HCC cells. By contrast, the selective knockdown of miRNA expression led to a significant increase in Casp- 8 levels and a marked reduction in programmed cell necrosis. Intriguingly, the sustained overexpression of Casp- 8 reversed the pro-necroptotic effects exerted by miRNA mimics. Finally, a strong inverse association between the level of miR-223 and the expression levels of nucleotide-binding oligomerization domain-like receptor family, pyrin domain-containing-3 inflammasome was observed in our HCC specimens. On the whole, the present study revealed a molecular link between the three-miRNA signature, comprising miR-371-5p, miR-373 and miR-543, and the negative necroptotic regulator Casp-8,
\end{abstract}

Correspondence to: Professor M. Aguennouz, Functional Genomics Laboratory, UOC Neurologia MNM, Department of Clinical and Experimental Medicine, University of Messina, Via Consolare Valeria 1, I-98125 Messina, Italy

E-mail: aguenoz@unime.it

Key words: necroptosis, hepatocellular carcinoma, microRNAs, caspase-8, inflammasome and presents evidence for its employment as a novel potential diagnostic, prognostic and therapeutic target in HCC.

\section{Introduction}

Hepatocellular carcinoma (HCC) is one of the most aggressive malignant tumors with respect to the disease course and mortality rate (1) Although numerous therapeutic strategies have been introduced, the prognosis of patients with HCC remains poor due to the frequent metastasis and recurrence $(2,3)$. Therefore, it is crucial to identify specific molecular targets and provide the basis for early diagnosis of HCC and targeted therapy, in order to achieve optimal outcomes and improve the quality of life and life expectancy of HCC patients.

MicroRNAs (miRNAs or miRs) are small, non-coding, single-stranded RNAs that regulate key cell processes, such as proliferation, differentiation and apoptosis (4-9), and exert major functional effects on various disorders, including numerous, if not all, cancers (10-13). Abnormalities in miRNA expression profiles may be caused by different mechanisms, such as amplifications, deletions, or sequence changes in the miRNA loci, epigenetic silencing and the dysregulation of transcription factors targeting specific miRNAs (14). Over the past decades, miRNAs have provided a powerful tool for health care intervention in a variety of tumor types (10-13). Increasing interest in the development of miRNAs as novel therapeutic biomarkers stems from the ability to interfere with miRNA levels through selective miRNA mimics or anti-miRs (15).

Recently, a number of miRNAs have been implicated as modulators of cell necroptosis $(16,17)$, a newly discovered pathway of regulated cell necrosis governed by receptorinteracting protein (RIP)1, RIP3 and mixed lineage kinase domain-like (MLKL) protein, which constitutes a critical cell-killing mechanism in response to severe stress, chemotherapy or inflammatory factors (18). Previous studies have demonstrated that necroptosis is associated with cancer initiation and progression, playing a dual and opposing role. Accordingly, while enhanced necroptosis concurs with cancer cell death, extensive cell death also increases the 
risk of proliferation and the metastasis of surviving cells by promoting the formation of reactive oxygen species, triggering the inflammatory process and inhibiting the immune response (19). In this regard, emphasis has been placed on the crucial role of certain miRNAs in the transcriptional regulation of the nucleotide-binding oligomerization domain-like receptor family, pyrin domain-containing-3 (NRLP3) inflammasome, an intracellular multiprotein complex that contributes to necroptosis upon the production of pro-inflammatory cytokines (20), with activity involved in the pathogenesis and progression of several cancers, including HCC $(21,22)$. In this context, recent investigations revealed that the caspase- 8 gene (Casp-8) is a key controller of NLRP3 activation (23-26); however, the mechanisms underlying this process have not yet been fully elucidated.

The aim of the present study was to identify potential interactions between a panel of miRNAs predicted by computational scoring as necroptotic pathway regulators and Casp- 8 in HCC specimens and cell lines. We identified three miRNAs, namely miR-371-5p, miR-373 and miR-543, which were upregulated in HCC tissues compared with adjacent non-cancerous tissues. These miRNAs have the ability to bind to their target Casp- 8 mRNA transcript in order to facilitate the necroptosis of HCC cells. Additionally, the data presented herein confirm the well-known association between miR-223 and NLRP3, as already reported in different disease contexts (27-31).

The findings of this study identified a key piece in the complex molecular puzzle of HCC, thereby providing new insight for a better understanding of the pathogenic mechanisms of this type of tumor. The assessment of the necroptotic pathway regulation by miRNAs depicts the starting point for a novel miRNA-based diagnostic, prognostic and therapeutic approach in HCC.

\section{Materials and methods}

Patient selection. This a retrospective study of tumor tissues from 38 patients with HCC treated by surgical resection at the University Hospital of Messina between 2011 and 2015. This study was approved by the local Institutional Review Board (IRB) of the University Hospital of Messina, Messina, Italy. The study protocol conforms to the ethical guidelines of the 1975 Declaration of Helsinki. Written informed consent was obtained from all subjects at the moment of the surgical procedures. For research purposes, no consent for retrospective de-identified data analysis was required as deemed by the IRB.

No patients received neoadjuvant therapy (radiotherapy and/or chemotherapy). The diagnosis of HCC was based on explant evaluation according to the revised WHO classification tumors (32). The clinical characteristics of the patients are presented in Table I.

Tissue samples. HCC tissues and their normal liver counterparts were frozen immediately after surgery and stored at $-80^{\circ} \mathrm{C}$ for biomolecular analyses.

Cells and cell culture. The Hep3B- $h$ CG cells were purchased from Sigma-Aldrich Italy (Milan, Italy) and maintained in Dulbecco's modified Eagle's medium (DMEM) with high glucose (HyClone, Logan, UT, USA) supplemented with $10 \%$ FBS. The hNHeps ${ }^{\mathrm{TM}}$ (human normal hepatocytes) cells were purchased from Lonza (Lonza, Walkersville, MD, USA) and maintained in hepatocyte culture medium (Lonza).

RNA extraction, cDNA synthesis and reverse transcriptionquantitative polymerase chain reaction ( $R T-q P C R)$. Total RNA was extracted from the HCC tissues and cell cultures using TRIzol reagent (Invitrogen/Thermo Fisher Scientific, Inc., Waltham, MA USA), according to the manufacturer's instructions. Total RNA concentration and integrity were examined using an Agilent Bioanalyzer (Agilent Technologies, Inc., Santa Clara, CA, USA). Subsequently, $300 \mathrm{ng}$ of total mRNA per sample was reverse transcribed into cDNA using the High Capacity cDNA Reverse Transcription kit (Applied Biosystems/Thermo Fisher Scientific, Inc.).

RT-qPCR for Casp-8 and NLRP3 expression was performed using a standard TaqMan PCR kit procedure on an AB-7300 RT-PCR system (Applied Biosystems/Thermo Fisher Scientific, Inc.) The reaction was performed with one cycle of $95^{\circ} \mathrm{C}$ for $5 \mathrm{~min}$ followed by 40 cycles at $95^{\circ} \mathrm{C}$ for $15 \mathrm{sec}$, $52^{\circ} \mathrm{C}$ for $20 \mathrm{sec}$ and $72^{\circ} \mathrm{C}$ for $30 \mathrm{sec}$. The mRNA levels of Casp- 8 and NLRP3 were normalized to endogenous $\beta$-actin (Applied Biosystems). Primers and probes were as previously reported $(33,34)$.

The control was represented by hNHeps ${ }^{T M}$ cells (described above) whose expression was considered as equal to 1. Relative fold expression and changes were calculated using the $2^{-\Delta \Delta C q}$ method (35).

miRNA isolation, cDNA synthesis and qPCR analysis. miRNAs were extracted from the HCC tissues and cells using the miRVana Isolation kit (Ambion/Thermo Fisher Scientific, Inc.), following the manufacturer's instructions. The enriched miRNA fraction was converted in cDNA using the TaqMan MicroRNA Reverse Transcriptase kit (Applied Biosystems/ Thermo Fisher Scientific, Inc.). hsa-miR-137, hsa-miR-223, hsa-miR-324, hsa-miR-371-5p, hsa-miR-372, hsa-miR-373, hsa-miR-455, hsa-miR-543, hsa-miR-595, and hsa- miR-661 were predicted by computational scoring as necroptotic pathway regulators $(36,37)$.

For miRNA quantification, $2 \mu \mathrm{l}$ of cDNA was used for each specific miRNA TaqMan Small RNA assay (Applied Biosystems/Thermo Fisher Scientific, Inc.) according to the manufacturer's instructions. All reverse transcription reactions, including no-template controls and reverse transcription controls, were performed in triplicate. RNU6 small nuclear RNA was used to normalize miRNA expression levels owing to its claimed expression stability and its wide use as loading control in several published miRNA expression studies $(38,39)$. Relative fold expression and changes were calculated using the $2^{-\Delta \Delta \mathrm{Cq}}$ method (35).

Target prediction tools. miRNA/Casp- 8 mRNA interactions were identified by means of bioinformatics algorithms that predict miRNA target sites (40). Specifically, three online databases, microRNA.org (www.microrna.org), miRDB (http://mirdb.org/miRDB/), and TargetScan (www.targetscan. org), were used. 
Table I. Clinical characteristics of the patients with HCC.

\begin{tabular}{lc}
\hline Sex (male/female) & $27 / 11$ \\
Mean age (range, years) & $68 \pm 11$ \\
HBsAg/Anti-HCV/NBNC & $8 / 13 / 17$ \\
Child-Pugh grading (A/B/C) & $21 / 17 / 0$ \\
Stage (I/II/IIIA/IIIB/IIIC/IV) & \\
0/26/12/0/0/0 & \\
Total bilirubin (mg/dl) & $44 \pm 10$ \\
Albumin (g/dl) & $3.5 \pm 0.4$ \\
PT (INR) & $4 \pm 0.8$ \\
Ascites (-/+) & $20 / 18$ \\
\hline
\end{tabular}

HBsAg, hepatitis B surface antigen; anti-HCV, antibodies against hepatitis C; NBNC, non-B non-C hepatitis.

Oligonucleotide transfection. hsa-miR-371-5p, hsa-miR-373 and hsa-miR-543 mimics/inhibitors (Qiagen, Milan, Italy) were transfected into the Hep3B-hCG cells using HiPerFect according to the manufacturer's instructions (Qiagen). The cells were transfected twice with 100 pmol of oligonucleotide per well $\left(0.5 \times 10^{6}\right.$ cells $)$ at 24 -h intervals. The transfected cells were assayed $48 \mathrm{~h}$ after the second transfection.

Plasmid constructs and transient transfection. The plasmid containing the full-length Casp- 8 cDNA was obtained as previously described (33). The 3'-UTR of Casp-8 and the mutation sequences were amplified by PCR using the primers with a $B g l$ II restriction site on each $5^{\prime}$ or $3^{\prime}$ strand. The PCR products were inserted into the $B g l \mathrm{II}$ sites of the pGL3-control vector (Promega, Madison, WI, USA) and identified by DNA sequencing.

The wild-type plasmids were created containing the 3'UTR of Casp- 8 with the complementary sequence of miR-371-5p (pGL3-Casp-8-3'UTR wild 1), miR-373 (pGL3-Casp-8-3'UTR wild 2) and miR-543 (pGL3-Casp-8-3'UTR wild 3). Three mutant plasmids were generated without the complementary sequence of miR-371-5p (pGL3-Casp-8-3'UTR mut 1), miR-373 (pGL3-Casp-8-3'UTR mut 2) and miR-543 (pGL3Casp-8-3'UTR mut 3).

For the luciferase reporter assay, the cells were seeded on 24-well plates and co-transfected using Lipofectamine 2000 (Invitrogen/Thermo Fisher Scientific, Inc.) with 100 ng per well of the resulting luciferase UTR-report vector, $2 \mathrm{ng}$ per well of pRLCMV vector (internal control, Promega), and $20 \mathrm{ng}$ per well of miR-371-5p, miR-373 and miR-543 mimics or inhibitors following the manufacturer's instructions (Qiagen). After $24 \mathrm{~h}$, the cells were lysed, and the relative luciferase activity was assessed with the Dual-Luciferase Assay Reporter System (Promega).

Necroptosis assay. The Hep3B- $h$ CG cells $\left(2 \times 10^{3}\right.$ cells/well) were pre-treated with the pan-caspase inhibitor, Z-VAD $(10 \mu \mathrm{M})$, for $1 \mathrm{~h}$ in order to induce RIPK3-dependent necroptosis, in the presence or absence of miRNA mimics and inhibitors. Tumor necrosis factor (TNF)- $\alpha(50 \mathrm{ng} / \mathrm{ml})$ was then added and the cells were incubated for an additional
$72 \mathrm{~h}$. Cell viability was assessed by MTT assay. In brief, $20 \mu \mathrm{l}$ of $5 \mathrm{mg} / \mathrm{ml}$ stock solution of 3-(4,5-dimetylthiazol-2-yl)2,5-diphenyltetrazolium bromide (Sigma-Aldrich) was added to each well, and the cells were incubated for $1 \mathrm{~h}$ at $37^{\circ} \mathrm{C}$ in a humidified atmosphere of $5 \% \mathrm{CO}_{2}$. The supernatants were carefully removed, and $100 \mu \mathrm{l}$ of DMSO (vehicle) was added to each well. The plates were subsequently shaken for $20 \mathrm{~min}$ at room temperature, and the absorbance of the solution was measured at $570 \mathrm{~nm}$. The absorbance of the untreated cells was set as $100 \%$ and cell survival was expressed as a percentage of this value.

Statistical analysis. Statistical analysis was performed using INSTAT, version 3.0 and PRISM version 4.0 software (GraphPad, San Diego, CA, USA). One-way analysis of variance (ANOVA), followed by a pair-wise multiple comparison test (Bonferroni correction), was performed to identify the differences among the groups. The association between Casp- 8 mRNA expression levels and clinicopathological characteristics was evaluated using Pearson's Chi-square test. Pearson's correlation analysis was used to estimate the correlation between the Casp- 8 mRNA levels and the miR-371-5p, miR-373 and miR-543 expression profiles, as well as between NLRP3 abundance and miR-223 expression. A value of $\mathrm{P}<0.05$ was considered to indicate a statistically significant difference.

\section{Results}

Expression of Casp-8 in HCC samples. Casp-8 is a cysteine protease that is a key regulator of cell apoptosis; however, its functional significance as a negative regulator of programmed cell necrosis has attracted growing attention over the years (41-43). Since different programmed cell death pathways, including necroptosis, have been recently implicated in hepatocarcinogenesis (44), in this study, we addressed the question of whether Casp- 8 plays a role in the programmed necrotic death of HCC cells. The values for Casp- $8 \mathrm{mRNA}$ levels were first determined in the normal liver (NL) samples in order to determine the cut-off point for Casp- 8 expression levels in the HCC samples. Casp- 8 expression levels (as defined by the ratio between the values measured in NL samples over those of hNHeps $^{\mathrm{TM}}$ cells) were between 0.95 and 1.6 (mean, 1.29 \pm 0.16 ). Values $\leq 0.81$ (determined as the mean minus 3 s.d.) were considered to represent the underexpression of Casp-8. As shown in Fig. 1A, a marked decrease in the Casp- 8 mRNA level was found in 34/38 (89.5\%) HCC samples. Box plots showing the individual means and scores of Casp- 8 mRNA expression in the NL and HCC specimens are presented in Fig. 2B. Likewise, a marked decrease in Casp- 8 expression levels was observed in the HCC cell line, Hep3B- $h$ CG, compared with the normal hepatocyte cell line, hNHeps ${ }^{\mathrm{TM}}$ (Fig. 1C). In addition, in order to gain insight into the role of Casp- 8 in HCC, we analyzed the association between Casp- 8 expression and various patient characteristics, including gender, age, Child-Pugh grade and stage (Table II). No significant association was observed between Casp- 8 expression and the clinical variables considered $(\mathrm{P}>0.05)$.

Prediction of miRNAs regulating necroptotic cell death that target Casp-8. To identify the potential upstream 


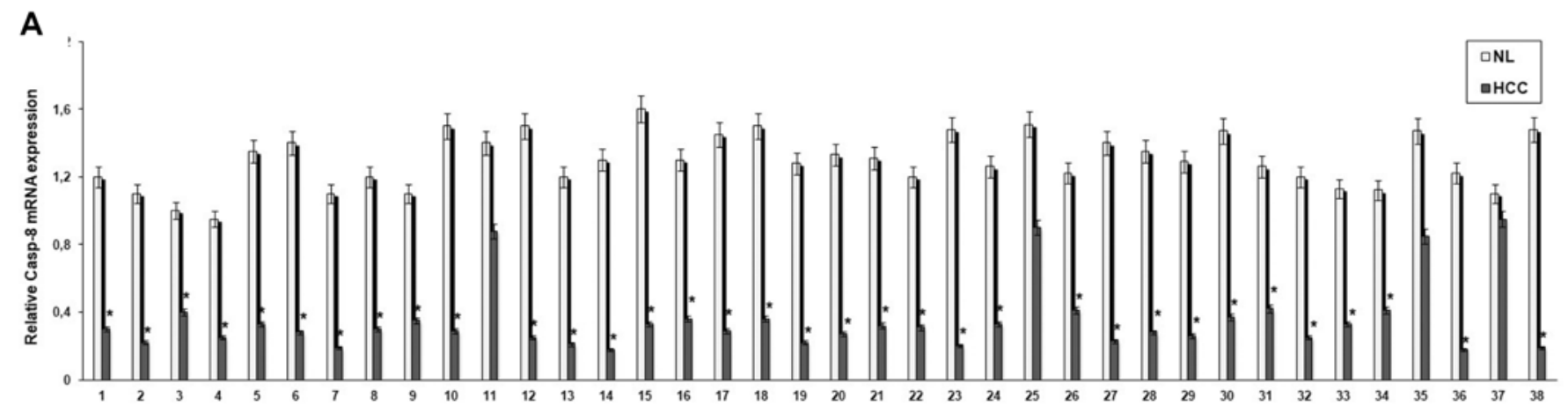

B

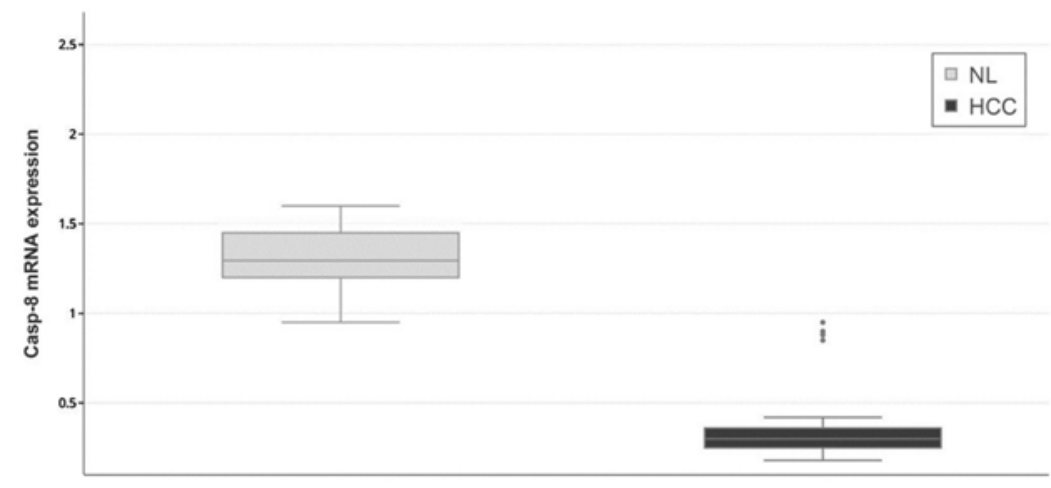

C

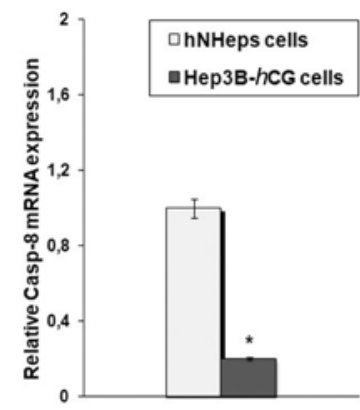

Figure 1. Expression levels of caspase-8 (Casp-8) in hepatocellular carcinoma (HCC) tissues and cells. (A) Total RNA, extracted from 38 HCC and paired normal liver (NL) tissues, was reverse-transcribed and analyzed by quantitative polymerase chain reaction (qPCR). The mRNA levels of $C a s p-8$ were normalized to the housekeeping gene, $\beta$-actin, as an internal control. Data are presented as the means \pm standard deviation of 3 independent experiments. ${ }^{*} \mathrm{P}<0.001$, compared to NL specimens. (B) Box plots relative to Casp- 8 mRNA expression in HCC and NL tissues. (C) Total RNA, extracted from the human HCC cell line, Hep3B- $h$ CG, and human normal hepatocytes $\left(\mathrm{hNHeps}^{\mathrm{TM}}\right)$, was reverse transcribed and analyzed by qPCR. The mRNA levels of Casp- 8 were normalized by using the housekeeping gene $\beta$-actin as internal control. Data are presented as the means \pm standard deviation of 3 independent experiments. "P $<0.001$, compared to the $\mathrm{hNHeps}{ }^{\mathrm{TM}}$ cells.

Table II. Association between Casp-8 expression and clinicopathological characteristics.

\section{Casp-8 mRNA expression}

Variables $\quad$ Low (n-34) $\quad$ High $(n=4) \quad$ P-value

\begin{tabular}{llll}
\hline Sex & & & 0.854 \\
Male & 24 & 3 & \\
Female & 10 & 1 & \\
Age (years) & & & 0.911 \\
$\quad \leq 60$ & 16 & 2 & \\
$\geq 60$ & 18 & 2 & \\
Child-Pugh grading & & & 0.401 \\
A & 18 & 3 & \\
B & 16 & 1 & \\
Stage & & & 0.765 \\
II & 23 & 3 & \\
IIIA & 11 & 1 & \\
\end{tabular}

executioners of Casp- 8 signaling in necrotic cascades, we first explored the expression profiles of a panel of miRNAs predicted by computational scoring as necroptotic pathway regulators $(36,37)$. Significantly higher levels of hsa-miR-137, hsa-miR-324, hsa-miR-371-5p, hsa-miR-372, hsa-miR-373, hsa-miR-455, hsa-miR-543, hsa-miR-595 and hsa- miR-661, and lower levels of miR-223, were observed in the HCC tissues compared with the NL specimens (Fig. 2A). A similar expression pattern of this miRNA panel was noted in the HCC cell line, Hep3B- $h$ CG, compared with the normal hepatocyte cell line, hNHeps ${ }^{\mathrm{TM}}$ (Fig. 2B). Subsequently, among these miRNAs, we searched for those that may have seed regions able to bind to the 3'UTR of Casp- 8 by using predictive bioinformatics programs (microRNA.org, miRDB and TargetScan). We identified miR-371-5p, miR-373 and miR-543 as potential putative Casp-8-targeting miRNAs. Of note, Pearson's correlation analysis revealed a strong negative correlation between the miR-371-5p, miR-373 and miR-543 expression profiles and Casp- 8 mRNA levels in the paired HCC tumor tissues $(\mathrm{r}=-0.689$ for $\mathrm{miR}-371-5 \mathrm{p} ; \mathrm{r}=-0.611$ for $\mathrm{miR}-373$; and $\mathrm{r}=-0.606$ for miR-543).

$m i R-371-5 p, m i R-373$ and miR-543 participate in the regulation of Casp- 8 expression. To validate the direct interaction of miR-371-5p, miR-373 and miR-543 with Casp- 8 mRNA (Fig. 3A), wild-type plasmids containing the 3'UTR of Casp-8 with complementary sequence of miR-371-5p (pGL3-Casp-83'UTR wild 1), miR-373 (pGL3-Casp-8-3'UTR wild 2) and miR-543 (pGL3-Casp-8-3'UTR wild 3) and the mutant 
A
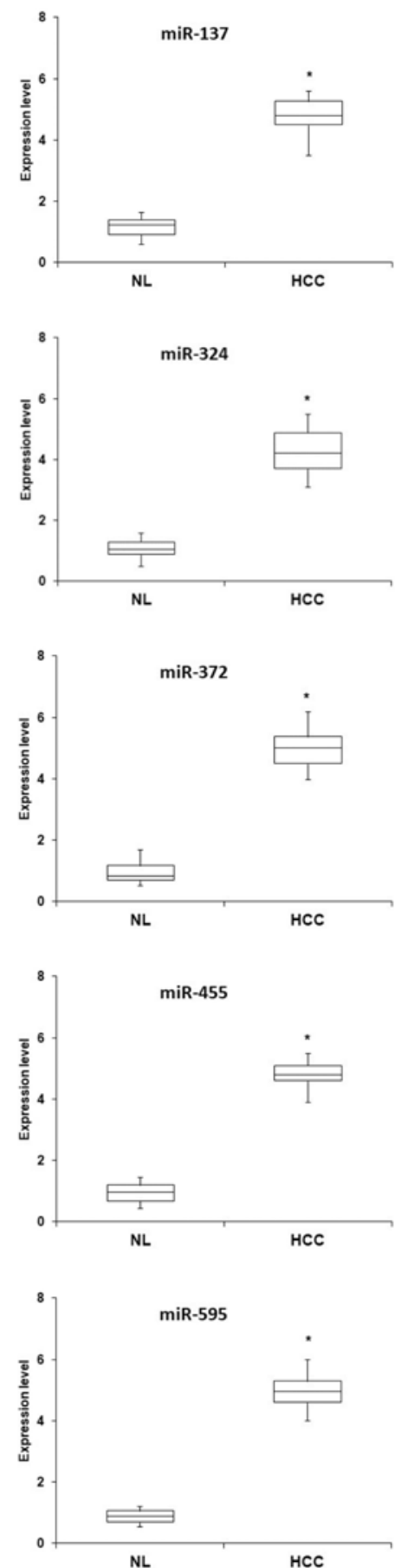
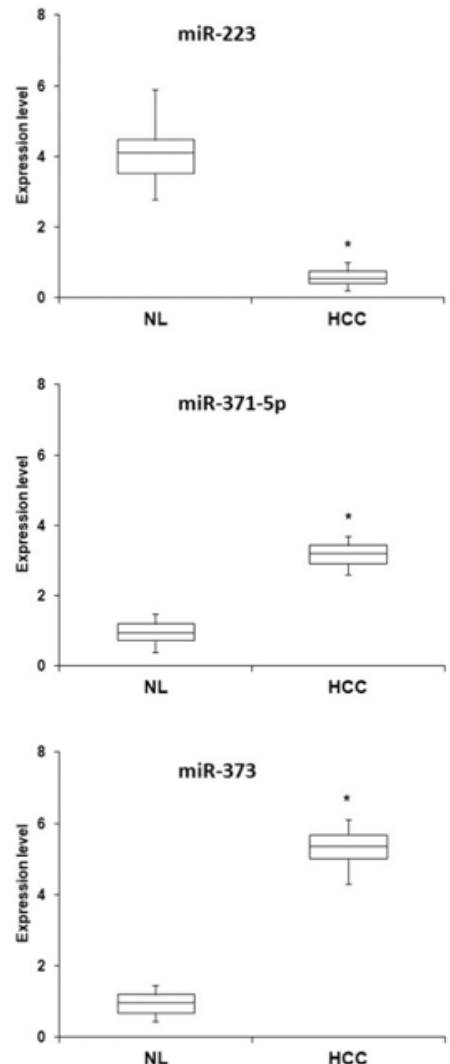

NL
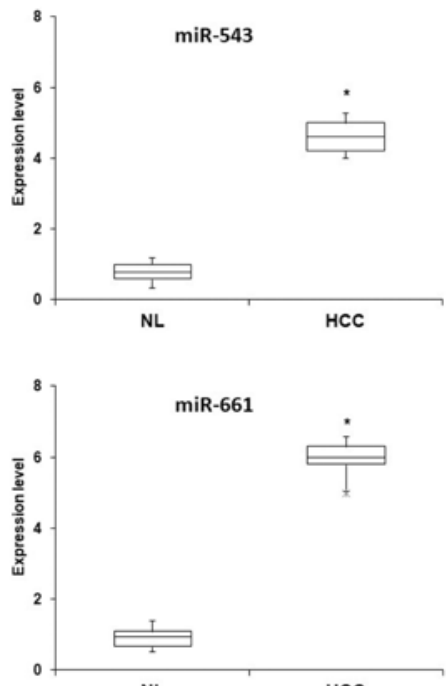

$\mathrm{HCC}$

B

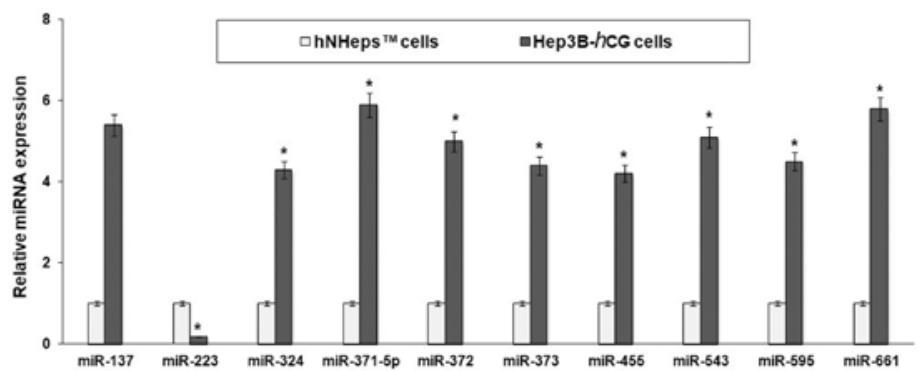

Figure 2. Expression profiles of a panel of miRNAs implicated in programmed cell necrosis in hepatocellular carcinoma (HCC) tissues and cells. (A) Total RNA was extracted from $38 \mathrm{HCC}$ and the paired normal liver (NL) tissues, reverse transcribed and analyzed by quantitative polymerase chain reaction (qPCR) to determine the amounts of miR-137, miR-223, miR-324, miR-371-5p, miR-372, miR-373, miR-455, miR-543, miR-595 and miR-661. Box plots showing means and scores of miRNA expression in NL and HCC specimens are shown. Data are representative of three independent experiments. (B) Total RNA, extracted from the human HCC cell line Hep3B- $h$ CG and human normal hepatocytes (hNHeps ${ }^{\mathrm{TM}}$ ), was reverse transcribed and analyzed by qPCR to determine the relative amounts of miR-137, miR-223, miR-324, miR-371-5p, miR-372, miR-373, miR-455, miR-543, miR-595 and miR-661. Data are presented as the means \pm standard deviation of 3 independent experiments. ${ }^{*} \mathrm{P}<0.001$, compared to the hNHeps ${ }^{\mathrm{TM}}$ cells. $^{\circ}$ 
A
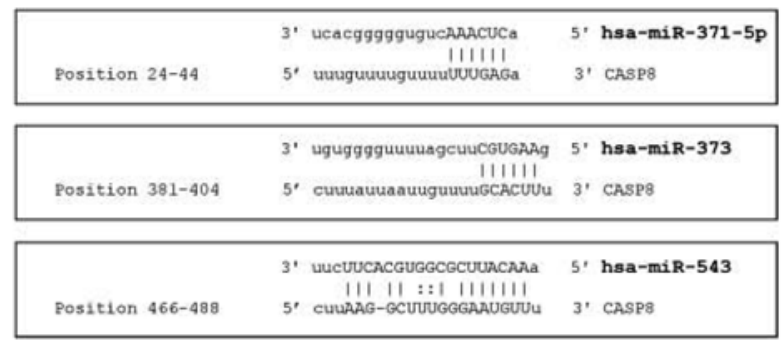

\section{B}
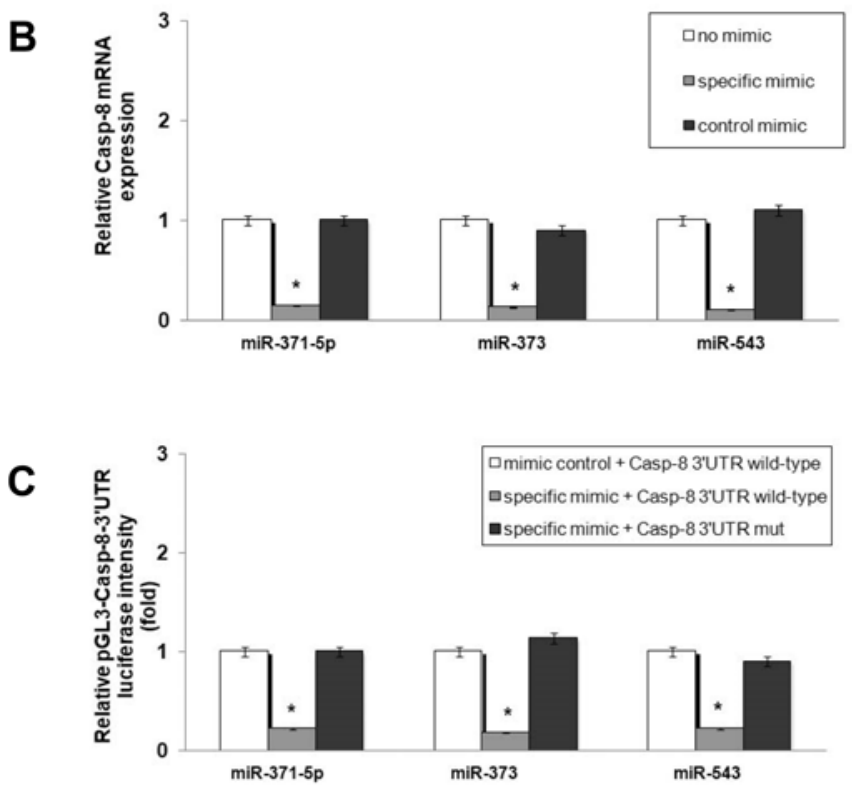

D

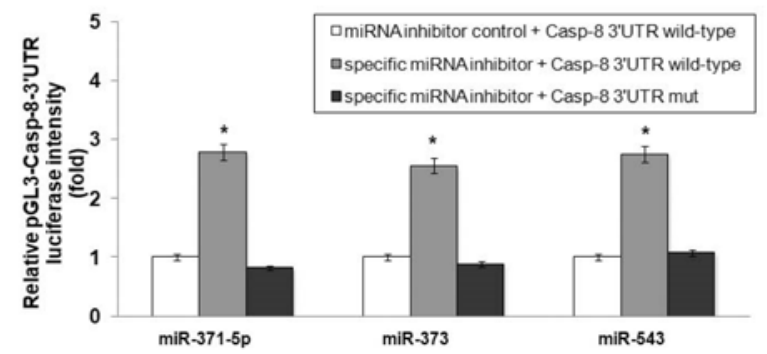

Figure 3. Caspase-8 (Casp-8) is the direct target of miR-371-5p, miR-373 and miR-543. (A) Schematic representation of the predicted interaction of miR-371-5p, miR-373 and miR-543 with Casp-8 3'UTR. (B) The mRNA levels of Casp- 8 were normalized by using the housekeeping gene $\beta$-actin as internal control. Data are presented as the means \pm standard deviation of 3 independent experiments. ${ }^{*} \mathrm{P}<0.001$, compared to untransfected cells. (C) Luciferase reporter assay was performed to detect the effect of individual miRNA mimics on the luciferase intensity controlled by 3 'UTR of Casp-8. Data are presented as the means \pm standard deviation of 3 independent experiments. ${ }^{*} \mathrm{P}<0.001$, compared to cells transfected with the mimic control. (D) Luciferase reporter assay was performed to detect the effect of individual miRNA inhibitors on the luciferase intensity controlled by 3'UTR of Casp-8. Data are presented as the means \pm standard deviation of 3 independent experiments. ${ }^{~} \mathrm{P}<0.001$, compared to cells transfected with the inhibitor control UTR, untranslated region.

plasmids without complementary sequence of miR-371-5p (pGL3-Casp-8-3'UTR mut 1), miR-373 (pGL3-Casp-83'UTR mut 2) and miR-543 (pGL3-Casp-8-3'UTR mut 3) were co-transfected into the Hep3B- $h$ CG cells together with specific mimics or inhibitors. We found that the enforced expression of miR-371-5p, miR-373 and miR-543 induced a marked decrease in Casp- 8 expression (Fig. 3B), accom-

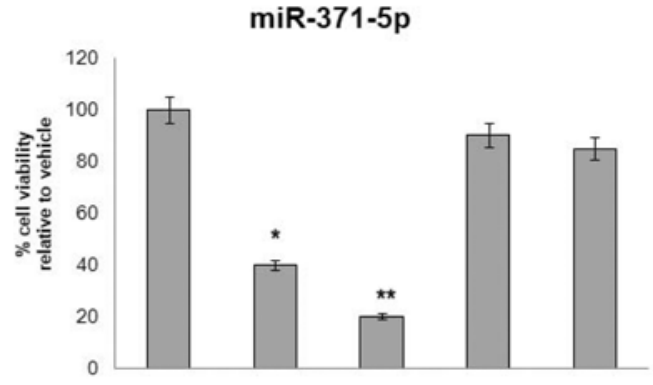

$\operatorname{miR}-373$

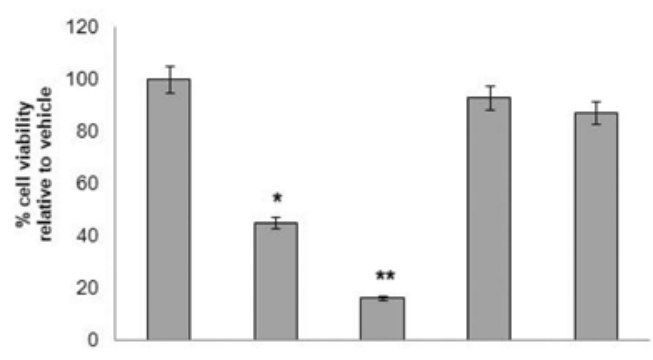

miR-543

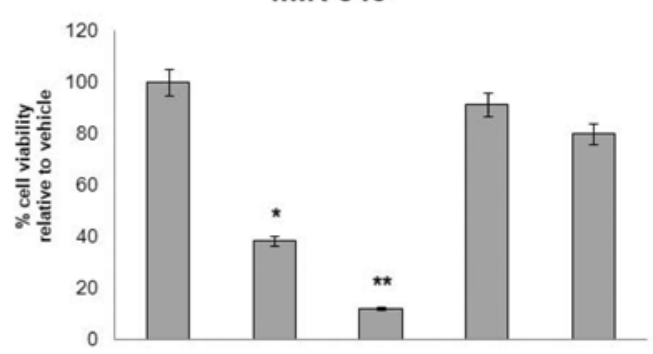

\begin{tabular}{|l|c|c|c|c|c|}
\hline Vehicle & + & + & + & + & + \\
\hline zVAD/TNF & - & + & + & + & + \\
\hline miRNA mimic & - & - & + & - & + \\
\hline miRNA inhibitor & - & - & - & + & - \\
\hline Casp-8 expression vector & - & - & - & - & + \\
\hline
\end{tabular}

Figure 4. miR-371-5p, miR-373, and miR-543 regulated the necroptosis of hepatocellular carcinoma (HCC) cells by targeting caspase-8 (Casp-8). Hep3B- $h$ CG cells were pre-treated with the pan-caspase inhibitor, zVAD $(10 \mu \mathrm{M})$ for $1 \mathrm{~h}$, then TNF- $\alpha(50 \mathrm{ng} / \mathrm{ml})$ was added followed by incubation for $72 \mathrm{~h}$. Where indicated, the cells were transiently transfected with Casp- 8 expression plasmid. Cell viability was assessed by MTT assay. ${ }^{*} \mathrm{P}<0.01$ and ${ }^{* *} \mathrm{P}<0.001$, compared to zVAD/TNF-treated cells.

panied by a significant decrease in the luciferase activity of pGL3-Casp-8-3'UTR wild-type plasmids (Fig. 3C; P<0.001), whereas the transcription of the pGL3-Casp-8-3'UTR mut vectors was not affected (Fig. 3C). Upon blocking the expression of the three miRNAs with specific inhibitors, luciferase intensity markedly increased in cells transfected with the pGL3-Casp-8-3'UTR wild-type vectors $(\mathrm{P}<0.001)$. No change was observed in the cells transfected with the pGL3Casp-8-3'UTR mut plasmids (Fig. 3D). These results provide evidence of Casp-8 as a target gene of miR-371-5p, miR-373 and miR-543 in our setting.

miR-371-5p, miR-373 and miR-543 regulated necroptosis of HCC cells by targeting Casp-8. Only a limited number of studies to date have reported the mechanisms through which miRNAs regulate necroptotic cell death $(16,17)$. Of note, miR-874 has been shown to enhance necroptosis by targeting Casp-8 (45). Hence, given the functional interaction of 


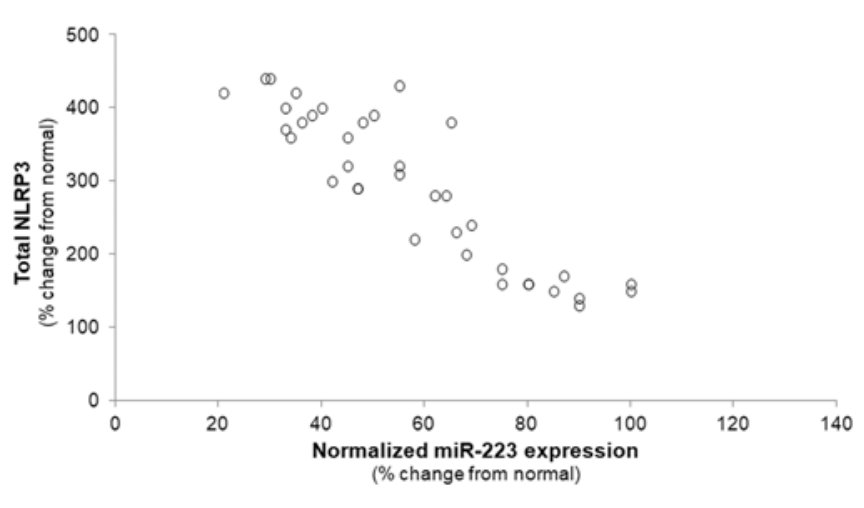

Figure 5. Inverse correlation between NLRP3 abundance and miR-223 expression in hepatocellular carcinoma samples. Total RNA was extracted from 38 hepatocellular carcinomas, reverse transcribed and analyzed by qPCR to determine the relative amounts of $N L R P 3$ and miR-223. Data were analyzed using Pearson's correlation analysis.

miR-371-5p, miR-373 and miR-543 with Casp-8, in this study, we attempted to elucidate whether these miRNAs play a role in the necrotic cascade orchestrated by Casp-8. Hep3B- $h \mathrm{CG}$ cells were first pre-treated with the pan-caspase inhibitor, Z-VAD $(10 \mu \mathrm{M})$, in the presence or absence of miRNA mimics and inhibitors, and then exposed to TNF- $\alpha$. The enforced expression of miR-371-5p, miR-373 and miR-543 markedly increased Z-VAD/TNF- $\alpha$-induced cell necroptosis, whereas the knockdown of miR-371-5p, miR-373 and miR-543 led to a marked reduction of programmed cell necrosis. Of note, the pro-necroptotic effects exerted by the miRNA mimics were abolished when Casp- 8 was ectopically overexpressed, thus indicating that this three-miRNA signature was able to regulate the necroptosis of Hep3B- $h$ CG cells via targeting Casp- 8 (Fig. 4).

Inverse association between miR-223 and NLRP3 in HCC samples. The miR-223-dependent post-transcriptional regulation of the NLRP3 inflammasome has been well validated in a variety of cell types $(27,30)$; however, this functional link has not been established yet in HCC. Therefore, in this study, we proceeded to investigate whether an inverse correlation between miR-223 abundance and NLRP3 mRNA expression also exists in HCC. A significant decrease in the miR-223 level was accompanied by a corresponding increase in NLRP3 in the HCC samples compared with the NL specimens ( $r=-0.3455$, $\mathrm{P}<0.01$ ), supporting the hypothesis that miR-223 likely also targets NLRP3 in HCC (Fig. 5).

\section{Discussion}

Necroptosis has recently attracted increasing attention as a newly identified type of programmed cell death that occurs when cells are subjected to severe stress or are exposed to chemotherapeutic agents or inflammatory factors (46-49). This type of regulated necrosis has been implicated in the pathogenesis of a variety of human diseases, such as neurodegeneration, ischemic reperfusion injury, Gaucher's disease, progressive atherosclerotic lesions, TNF-mediated hypothermia and systemic inflammation (50). However, the role of necroptosis in cancer remains controversial, as some authors consider this phenomenon a double-edged sword (19). Indeed, although a number of studies have reported that necroptosis prevents the onset and progression of cancer and promotes its therapy by proving a plethora of cancer cell lines highly sensitive and responsive to necroptosis inducers (18), emerging data have revealed that necroptosis may in fact contribute to cancer development (51-53). The rapid and extensive release of damage-associated molecules [e.g., interleukin (IL)-1 family cytokines] from necroptotic cancer cells may act as a potent inducer of inflammation (54), which in turn leads to tumorigenesis through the stimulation of angiogenesis and metastasis, as well as to the mitigation of the adaptive immune responses $(55,56)$. miRNAs were recently identified as new participants in regulated necrosis (57); however, the underlying mechanisms have not yet been fully elucidated. The findings of the present study indicated that a novel three-miRNA signature, including miR-371-5p, miR-373 and miR-543, is critically involved in programmed necroptotic death of HCC cells. This assertion was strongly supported by functional experiments in which enforced expression of these miRNAs markedly increased Z-VAD/TNF- $\alpha$-induced necroptosis in Hep3B- $h$ CG cells, whereas their specific knockdown was able to significantly attenuate programmed necrosis (Fig. 4).

Indeed, among all the miRNAs initially predicted by computational scoring as necroptotic pathway regulators, we restricted our investigations to those targeting the necroptosis inhibitor Casp- 8 gene, i.e., miR-371-5p, miR-373 and miR-543. Consequently, since miR-137, miR-324, miR-371-5p, miR-372, miR-373, miR-455, miR-543, miR-595 and miR-661 did not present regions able to bind to the 3'UTR of Casp-8 according to the target prediction results, they were not included in our subsequent experimental analyses. However, these miRNAs will be the subject of our further (in-progress) studies aimed at defining other metabolic pathways involved in necroptosis.

From the results obtained by the luciferase assay experiments, we found that miR-371-5p, miR-373 and miR-543 suppressed the expression of Casp- 8 through direct interaction with the 3'UTR of the target (Fig. 3). Of note, emerging evidence indicates that Casp-8 is an important negative regulator of necroptosis (43). Treatment with Z-VAD, a pan-caspase inhibitor, was found to promote necroptosis, since Casp-8, when inactivated, cannot cleave the two key inducers of necroptosis, namely RIP1 and RIP3 $(43,58,59)$. Moreover, the pharmacological inhibition of Casp- 8 induces the autocrine production of TNF, which triggers necroptosis (60). The results reported herein may shed new light into the mechanisms regulating programmed necrosis in $\mathrm{HCC}$, revealing that the three miRNAs of interest induce necroptosis by suppressing Casp-8. Accordingly, we observed that the pro-necroptotic effects exerted by miRNA mimics were abolished when Casp- 8 was ectopically overexpressed (Fig. 4). Higher expression levels of miR-371-5p, miR-373 and miR-543 were significantly associated with lower amounts of Casp- 8 mRNA in HCC tissues compared with paired normal tissues (Figs. 1 and 2), thereby supporting the key role of these miRNAs in controlling Casp-8 expression.

Of note, miR-373 has been previously implicated in several types of cancers as an oncomiR (61), with its downregulation resulting in the growth arrest and the apoptosis of 
tumor cells (62). Moreover, the increased expression levels of miR-371-5p have been associated with a shorter survival and a poor prognosis of patients with pancreatic cancer (63). Similarly, miR-543 has been found to be closely associated with tumor size, TNM stage and metastasis, as well as with a poor overall and disease-free survival (64). In this context, our study may provide new insight into the necroptotic function of this three-miRNA signature, further providing valuable insights into its role in $\mathrm{HCC}$ and its value as a potential predictor of poor prognosis. Indeed, the present findings are in line with those of a recent study reporting that pro-necroptotic pathways promote inflammatory cytokine production, thereby sustaining tumor growth (51).

Another meaningful result of this study was the significant inverse correlation between the expression pattern of miR-223-3p and that of the NLRP3 inflammasome found in the resected tissue specimens from HCC patients (Fig. 5). Specifically, we demonstrated that the downregulation of miR-223 was closely associated with increased NLRP3 mRNA levels. This finding effectively supports the hypothesis that miR-223 acts as a negative regulator of NLRP3 inflammasome activity, as it was already observed in other pathological conditions $(27,29)$. It is well recognized that NLRP3 is an important mediator involved in cell necroptosis (30), and it has been suggested that its activation may be modulated by Casp-8 (23,26). Of note, a recent study demonstrated that miR-223-3p prevents the ischemia/reperfusion-induced cardiac necroptosis via targeting NLRP3 inflammasome signaling (17). Therefore, the data presented herein may uncover a novel role for miR-223-3p and its target gene, NLRP3, in the context of $\mathrm{HCC}$, strengthening the inextricable association between these molecular effectors involved in programmed cell necrosis. Defining the role of miR-223-3p-mediated NLRP3 signaling in these and other potential necroptotic models is mandatory for the better understanding the complex crosstalk regulating programmed cell death and for testing new disease-specific medical treatments.

In conclusion, the results from the present study may provide new insight into the complex miRNA-governed network of regulated necrosis, and suggest that the newly identified signature of necroptosis-regulating miRNAs may represent a promising diagnostic and prognostic biomarker as well an effective therapeutic target for HCC.

However, further studies are required to verify the interplay between these miRNAs, their target genes and HCC progression, and to validate our findings for applicability in the clinical setting.

\section{Acknowledgements}

Not applicable.

\section{Funding}

No funding was received.

\section{Availability of data and materials}

All data generated or analyzed during this study are included in this published article.

\section{Authors' contributions}

MV, FP, RMDG, MB and MA contributed to the conception and design of the study; $\mathrm{MB}, \mathrm{AB}$ and $\mathrm{GN}$ were involved in the selection of the biopsy tissues. FP, RO, RA and MA acquired the data; MV, FP and MA analyzed and interpreted the data; RMDG, MB and GN participated in the revision of the article. All authors have read and approved the final manuscript.

\section{Ethics approval and consent to participate}

This study was approved by the local Institutional Review Board (IRB) of the University Hospital of Messina, Messina, Italy. The study protocol conforms to the ethical guidelines of the 1975 Declaration of Helsinki. Written informed consent was obtained from all subjects at the moment of the surgical procedures. For research purposes, no consent for retrospective de-identified data analysis was required as deemed by the IRB.

\section{Consent for publication}

Not applicable.

\section{Competing interests}

The authors declare that they have no competing interests.

\section{References}

1. Jemal A, Bray F, Center MM, Ferlay J, Ward E and Forman D: Global cancer statistics. CA Cancer J Clin 61: 69-90, 2011.

2. Mlynarsky L, Menachem Y and Shibolet O: Treatment of hepatocellular carcinoma: Steps forward but still a long way to go. World J Hepatol 7: 566-574, 2015.

3. Tang ZY: Hepatocellular carcinoma - cause, treatment and metastasis. World J Gastroenterol 7: 445-454, 2001.

4. Herranz $\mathrm{H}$ and Cohen SM: MicroRNAs and gene regulatory networks: Managing the impact of noise in biological systems. Genes Dev 24: 1339-1344, 2010.

5. Arner P and Kulyté A: MicroRNA regulatory networks in human adipose tissue and obesity. Nat Rev Endocrinol 11: 276-288, 2015.

6. Fang S, Deng Y, Gu P and Fan X: MicroRNAs regulate bone development and regeneration. Int J Mol Sci 16: 8227-8253, 2015.

7. Kohlhapp FJ, Mitra AK, Lengyel E and Peter ME: MicroRNAs as mediators and communicators between cancer cells and the tumor microenvironment. Oncogene 34: 5857-5868, 2015.

8. Su Y, Wu H, Pavlosky A, Zou LL, Deng X, Zhang ZX and Jevnikar AM: Regulatory non-coding RNA: New instruments in the orchestration of cell death. Cell Death Dis 7: e2333, 2016.

9. Tran DDH, Kessler C, Niehus SE, Mahnkopf M, Koch A and Tamura T: Myc target gene, long intergenic noncoding RNA, Linc00176 in hepatocellular carcinoma regulates cell cycle and cell survival by titrating tumor suppressor microRNAs. Oncogene 37: 75-85, 2018.

10. Allegra A, Alonci A, Campo S, Penna G, Petrungaro A, Gerace D and Musolino C: Circulating microRNAs: New biomarkers in diagnosis, prognosis and treatment of cancer (Review). Int $\mathbf{J}$ Oncol 41: 1897-1912, 2012.

11. Kunej T, Godnic I, Ferdin J, Horvat S, Dove P and Calin GA: Epigenetic regulation of microRNAs in cancer: An integrated review of literature. Mutat Res 717: 77-84, 2011.

12. Zhang B, Pan X, Cobb GP and Anderson TA: microRNAs as oncogenes and tumor suppressors. Dev Biol 302: 1-12, 2007.

13. Mizuguchi Y, Takizawa T, Yoshida H and Uchida E: Dysregulated miRNA in progression of hepatocellular carcinoma: A systematic review. Hepatol Res 46: 391-406, 2016.

14. Croce CM: Causes and consequences of microRNA dysregulation in cancer. Nat Rev Genet 10: 704-714, 2009. 
15. Krützfeldt J, Rajewsky N, Braich R, Rajeev KG, Tuschl T, Manoharan M and Stoffel M: Silencing of microRNAs in vivo with 'antagomirs'. Nature 438: 685-689, 2005.

16. Afonso MB, Rodrigues PM, Simão AL, Gaspar MM, Carvalho T, Borralho P, Bañales JM, Castro RE and Rodrigues CMP: miRNA-21 ablation protects against liver injury and necroptosis in cholestasis. Cell Death Differ: Dec 11, 2017 (Epub ahead of print). doi: 10.1038/s41418-017-0019-x.

17. Qin D, Wang X, Li Y, Yang L, Wang R, Peng J, Essandoh K, Mu X, Peng T, Han Q, et al: MicroRNA-223-5p and -3p cooperatively suppress necroptosis in ischemic/reperfused hearts. J Biol Chem 291: 20247-20259, 2016.

18. Chen D, Yu J and Zhang L: Necroptosis: An alternative cell death program defending against cancer. Biochim Biophys Acta 1865 228-236, 2016.

19. Wang T, Jin Y, Yang W, Zhang L, Jin X, Liu X, He Y and Li X Necroptosis in cancer: An angel or a demon? Tumour Biol 39: 1010428317711539, 2017.

20. Sutterwala FS, Haasken S and Cassel SL: Mechanism of NLRP3 inflammasome activation. Ann N Y Acad Sci 1319: 82-95, 2014.

21. Davis BK, Wen H and Ting JP: The inflammasome NLRs in immunity, inflammation, and associated diseases. Annu Rev Immunol 29: 707-735, 2011.

22. Wei Q, Mu K, Li T, Zhang Y, Yang Z, Jia X, Zhao W, Huai W, Guo P and Han L: Deregulation of the NLRP3 inflammasome in hepatic parenchymal cells during liver cancer progression. Lab Invest 94: 52-62, 2014.

23. Gurung P, Anand PK, Malireddi RK, Vande Walle L, Van Opdenbosch N, Dillon CP, Weinlich R, Green DR, Lamkanfi $\mathrm{M}$ and Kanneganti TD: FADD and caspase- 8 mediate priming and activation of the canonical and noncanonical Nlrp3 inflammasomes. J Immunol 192: 1835-1846, 2014.

24. Weng D, Marty-Roix R, Ganesan S, Proulx MK, Vladimer GI, Kaiser WJ, Mocarski ES, Pouliot K, Chan FK, Kelliher MA, et al: Caspase- 8 and RIP kinases regulate bacteria-induced innate immune responses and cell death. Proc Natl Acad Sci USA 111: 7391-7396, 2014

25. Gurung $P$ and Kanneganti TD: Novel roles for caspase- 8 in IL-1 $\beta$ and inflammasome regulation. Am J Pathol 185: 17-25, 2015.

26. Kang TB, Yang SH, Toth B, Kovalenko A and Wallach D: Caspase- 8 blocks kinase RIPK3-mediated activation of the NLRP3 inflammasome. Immunity 38: 27-40, 2013.

27. Xie XJ, Ma LG, Xi K, Fan DM, Li JG, Zhang Q and Zhang W: Effects of microRNA-223 on morphine analgesic tolerance by targeting NLRP3 in a rat model of neuropathic pain. Mol Pain 13: $1744806917706582,2017$.

28. Neudecker V, Haneklaus M, Jensen O, Khailova L, Masterson JC, Tye H, Biette K, Jedlicka P, Brodsky KS, Gerich ME, et al: Myeloid-derived miR-223 regulates intestinal inflammation via repression of the NLRP3 inflammasome. J Exp Med 214: 1737-1752, 2017.

29. Yang Z, Zhong L, Xian R and Yuan B: MicroRNA-223 regulates inflammation and brain injury via feedback to NLRP3 inflammasome after intracerebral hemorrhage. Mol Immunol 65 : 267-276, 2015.

30. Bauernfeind F, Rieger A, Schildberg FA, Knolle PA, Schmid-Burgk JL and Hornung V: NLRP3 inflammasome activity is negatively controlled by miR-223. J Immunol 189 : 4175-4181, 2012

31. Haneklaus M, Gerlic M, Kurowska-Stolarska M, Rainey AA Pich D, McInnes IB, Hammerschmidt W, O'Neill LA and Masters SL: Cutting edge: miR-223 and EBV miR-BART15 regulate the NLRP3 inflammasome and IL-1 $\beta$ production. J Immunol 189: 3795-3799, 2012.

32. Louis DN, Ohgaki H, Wiestler OD, Cavenee WK, Burger PC, Jouvet A, Scheithauer BW and Kleihues P: The 2007 WHO classification of tumours of the central nervous system. Acta Neuropathol 114: 97-109, 2007

33. Venza M, Visalli M, Biondo C, Oteri R, Agliano F, Morabito S, Teti D and Venza I: Epigenetic marks responsible for cadmiuminduced melanoma cell overgrowth. Toxicol In Vitro 29: 242-250, 2015.

34. Vilaysane A, Chun J, Seamone ME, Wang W, Chin R, Hirota S, Li Y, Clark SA, Tschopp J, Trpkov K, et al: The NLRP3 inflammasome promotes renal inflammation and contributes to CKD. J Am Soc Nephrol 21: 1732-1744, 2010.

35. Livak KJ and Schmittgen TD: Analysis of relative gene expression data using real-time quantitative PCR and the $2(-\Delta \Delta \mathrm{C}(\mathrm{T}))$ Method. Methods 25: 402-408, 2001
36. Vlachos IS, Kostoulas N, Vergoulis T, Georgakilas G, Reczko M, Maragkakis M, Paraskevopoulou MD, Prionidis K, Dalamagas T and Hatzigeorgiou AG: DIANA miRPath v.2.0: Investigating the combinatorial effect of microRNAs in pathways. Nucleic Acids Res 40W: W498-W504, 2012

37. Warde-Farley D, Donaldson SL, Comes O, Zuberi K, Badrawi R, Chao P, Franz M, Grouios C, Kazi F, Lopes CT, et al: The GeneMANIA prediction server: Biological network integration for gene prioritization and predicting gene function. Nucleic Acids Res 38 (Suppl 2): W214-W220, 2010.

38. Peltier HJ and Latham GJ: Normalization of microRNA expression levels in quantitative RT-PCR assays: Identification of suitable reference RNA targets in normal and cancerous human solid tissues. RNA 14: 844-852, 2008.

39. Song J, Bai Z, Han W, Zhang J, Meng H, Bi J, Ma X, Han S and Zhang Z: Identification of suitable reference genes for qPCR analysis of serum microRNA in gastric cancer patients. Dig Dis Sci 57: 897-904, 2012

40. Krek A, Grün D, Poy MN, Wolf R, Rosenberg L, Epstein EJ, MacMenamin P, da Piedade I, Gunsalus KC, Stoffel M, et al: Combinatorial microRNA target predictions. Nat Genet 37: 495-500, 2005

41. Feng S, Yang Y, Mei Y, Ma L, Zhu DE, Hoti N, Castanares M and Wu M: Cleavage of RIP3 inactivates its caspase-independent apoptosis pathway by removal of kinase domain. Cell Signal 19: 2056-2067, 2007.

42. Günther C, Martini E, Wittkopf N, Amann K, Weigmann B, Neumann H, Waldner MJ, Hedrick SM, Tenzer S, Neurath MF, et al: Caspase- 8 regulates TNF- $\alpha$-induced epithelial necroptosis and terminal ileitis. Nature 477: 335-339, 2011

43. Oberst A, Dillon CP, Weinlich R, McCormick LL, Fitzgerald P, Pop C, Hakem R, Salvesen GS and Green DR: Catalytic activity of the caspase-8-FLIP(L) complex inhibits RIPK3-dependent necrosis. Nature 471: 363-367, 2011.

44. Vucur M, Reisinger F, Gautheron J, Janssen J, Roderburg C, Cardenas DV, Kreggenwinkel K, Koppe C, Hammerich L, Hakem R, et al: RIP3 inhibits inflammatory hepatocarcinogenesis but promotes cholestasis by controlling caspase-8- and JNK-dependent compensatory cell proliferation. Cell Reports 4: 776-790, 2013.

45. Wang K, Liu F, Zhou LY, Ding SL, Long B, Liu CY, Sun T, Fan YY, Sun L and Li PF: miR-874 regulates myocardial necrosis by targeting caspase-8. Cell Death Dis 4: e709, 2013.

46. Chan FK, Shisler J, Bixby JG, Felices M, Zheng L, Appel M, Orenstein J, Moss B and Lenardo MJ: A role for tumor necrosis factor receptor-2 and receptor-interacting protein in programmed necrosis and antiviral responses. J Biol Chem 278: 51613-51621, 2003.

47. Han W, Li L, Qiu S, Lu Q, Pan Q, Gu Y, Luo J and Hu X: Shikonin circumvents cancer drug resistance by induction of a necroptotic death. Mol Cancer Ther 6: 1641-1649, 2007.

48. Newton K and Manning G: Necroptosis and Inflammation. Annu Rev Biochem 85: 743-763, 2016.

49. Long JS and Ryan KM: New frontiers in promoting tumour cell death: Targeting apoptosis, necroptosis and autophagy. Oncogene 31: 5045-5060, 2012

50. Zhou W and Yuan J: Necroptosis in health and diseases. Semin Cell Dev Biol 35: 14-23, 2014.

51. Liu X, Zhou M, Mei L, Ruan J, Hu Q, Peng J, Su H, Liao H, Liu S, Liu W, et al: Key roles of necroptotic factors in promoting tumor growth. Oncotarget 7: 22219-22233, 2016.

52. Seifert L, Werba G, Tiwari S, Giao Ly NN, Alothman S, Alqunaibit D, Avanzi A, Barilla R, Daley D, Greco SH, et al: The necrosome promotes pancreatic oncogenesis via CXCL1 and Mincle-induced immune suppression. Nature 532: 245-249, 2016.

53. Strilic B, Yang L, Albarrán-Juárez J, Wachsmuth L, Han K Müller UC, Pasparakis M and Offermanns S: Tumour-cellinduced endothelial cell necroptosis via death receptor 6 promotes metastasis. Nature 536: 215-218, 2016.

54. Pasparakis $M$ and Vandenabeele P: Necroptosis and its role in inflammation. Nature 517: 311-320, 2015.

55. Mantovani A, Allavena P, Sica A and Balkwill F: Cancer-related inflammation. Nature 454: 436-444, 2008.

56. Korniluk A, Koper O, Kemona H and Dymicka-Piekarska V: From inflammation to cancer. Ir J Med Sci 186: 57-62, 2017.

57. Su Z, Yang Z, Xu Y, Chen Y and Yu Q: MicroRNAs in apoptosis, autophagy and necroptosis. Oncotarget 6: 8474-8490, 2015.

58. Geserick P, Hupe M, Moulin M, Wong WW, Feoktistova M, Kellert B, Gollnick H, Silke J and Leverkus M: Cellular IAPs inhibit a cryptic CD95-induced cell death by limiting RIP1 kinase recruitment. J Cell Biol 187: 1037-1054, 2009. 
59. Welz PS, Wullaert A, Vlantis K, Kondylis V, FernándezMajada V, Ermolaeva M, Kirsch P, Sterner-Kock A, van Loo G and Pasparakis M: FADD prevents RIP3-mediated epithelial cell necrosis and chronic intestinal inflammation. Nature 477: 330-334, 2011.

60. Hitomi J, Christofferson DE, Ng A, Yao J, Degterev A, Xavier RJ and Yuan J: Identification of a molecular signaling network that regulates a cellular necrotic cell death pathway. Cell 135: $1311-1323,2008$

61. Huang Q, Gumireddy K, Schrier M, le Sage C, Nagel R, Nair S, Egan DA, Li A, Huang G, Klein-Szanto AJ, et al: The microRNAs $\mathrm{miR}-373$ and $\mathrm{miR}-520 \mathrm{c}$ promote tumour invasion and metastasis. Nat Cell Biol 10: 202-210, 2008.
62. Stelzer Y, Sagi I and Benvenisty N: Involvement of parental imprinting in the antisense regulation of onco-miR-372-373. Nat Commun 4: 2724, 2013.

63. He D, Miao H, Xu Y, Xiong L, Wang Y, Xiang H, Zhang H and Zhang Z: MiR-371-5p facilitates pancreatic cancer cell proliferation and decreases patient survival. PLoS One 9: e112930, 2014.

64. Zhai F, Cao C, Zhang L and Zhang J: miR-543 promotes colorectal cancer proliferation and metastasis by targeting KLF4. Oncotarget 8: 59246-59256, 2017. 